\title{
Short-term Reactive Power Reserve Optimization Based on Trajectory Sensitivity
}

\author{
Quancai Sun ${ }^{\dagger}$, Haozhong Cheng*, Jian Zhang**, Baiqing Li** and Yue Song***
}

\begin{abstract}
An increasing concern is paid to short-term voltage stability with the growth of penetration of induction motor loads. Reactive power reserve(RPR) of power system is critical to improve voltage stability. A definition of short-term voltage stability-related RPR(SVRPR) is proposed. Generators vary their contributions to voltage stability with their location and system condition, etc. Voltage support coefficient based on the second-order trace sensitivity method is proposed to evaluate SVRPR's contribution to short-term voltage stability. The evaluation method can account for the generator's reactive support in transient process and the contingency severity. Then an optimization model to improve short-term voltage stability is built. To deal with multiple contingencies, contingency weight taking into account both its probability and severity is proposed. The optimization problem is solved by primal dual interior point method. Testing on IEEE_39 bus system, it is indicated that the method proposed is effective. Short-term voltage stability is improved significantly by the way of SVRPR optimization. Hence, the approach can be used to prevent the happening of voltage collapse during system's contingency.
\end{abstract}

Keywords: Reactive power reserve, Short-term voltage stability, Contingency, Optimization

\section{Introduction}

The main contribution of RPR is maintaining voltage security when all kinds of disturbances occur in power system. Generally speaking, voltage collapse will happen when reactive power demand of power system can't be satisfied because of restrictions of var sources output capability and network transmission. To avoid voltage collapse or voltage dip, it is necessary to preserve enough RPR in power system [1]. Appropriate RPR profile can be obtained by proper reactive power management. Hence, RPR optimization by reactive power dispatch is very significant to operators to improve voltage security.

However, a generator's reactive support has different properties in its short- and long-term dynamics because the response of the generator's automatic excitation system is different when considering different durations [2]. Considering short-term dynamics, a generator can begin a forced excitation and produce large amounts of reactive power to support a drop in system voltage; thus, we cannot neglect the forced excitation response in this case. Considering long-term dynamics, the RPR supply is primarily restricted by the rated excitation, and thus, the most important factor is the reactive power that can be

$\dagger$ Corresponding Author: Dep. of Electrical Engineering, Shanghai Jiao Tong University, China. (qcsun@sjtu.edu.cn)

* Dep. of Electrical Engineering, Shanghai Jiao Tong University, China. (chenghz@sjtu.edu.cn)

** China Electric power Research Institute, China. ( \{jianzhang, libq\}@epri.sgcc.com.cn)

*** Dep of Electrical and Electronics Engineering, The University of Hong Kong, Hong Kong SAR, China. (yuesong@eee.hku.hk)

Received: April 11, 2016; Accepted: December 24, 2016 provided over the long-term. Therefore, the RPR concerning short- and long-term voltage stabilities should be separately defined and examined.

There have been some studies on long-term voltage stability-related RPR(LV-RPR). Definitions of generator RPR can be classified into two categories: technical RPR $[3,4]$ and effective RPR $[5,6]$. However voltage stability depends on system RPR which is based on all of the RPRs in the system. There are usually two ways to evaluate system RPR. One is simply adding all generator effective reactive reserves up as system RPR [7]. The other one is summing generator technical reactive reserves with weighting factors $[8,9]$. The latter one has been draw more attention since it can put it into consideration that the contribution of RPR to voltage stability varies with the generator location. Based on the above system reactive reserves, many LVRPR optimization methods have been proposed $[8,10]$. But the accuracy and applicability of the optimization model still need to be improved.

The aforementioned research on RPR evaluation and optimization are concerned with long-term or static voltage stability, while so far no RPR definitions directly related to short-term stability has been proposed. Evaluation of RPR with respect to contingencies were proposed, but just on quasi-steady state time scale $[3,11]$. However, along with large scale and long distance transmission system's input as well as increasing proportion of wind farm and motor in power system, short-term voltage security is becoming a predominant problem [12]. A clustering based method to group dynamic contingencies is proposed and an approach 
to identify dynamic voltage control area and the most effective candidate locations for placing dynamic reactive sources [13]. However, large amount of information from contingencies considering various system scenarios are needed in this approach, and the results are more suitable for dynamic reactive source planning. Hence it is urgent to present the definition and evaluation of SVRPR to aid the system operator in operation.

To assess SVRPR' contributions to short-term voltage stability during contingency, dynamic information of power system must be considered, especially for var sources such as generators. Time domain simulation is welladapted to component models and can reflect component dynamic characteristics comprehensively. In recent years, researchers have done heuristic works of applying trace sensitivity to study power system's stability [14]. Most of the researches focus on the first-order trajectory sensitivity. However, a power system is a complex nonlinear system. When the system suffering large disturbances, the firstorder trajectory sensitivity cannot reflect control variables' effect to state variables accurately [15].

The research of reactive power reserves with respect to contingencies has been studied in [3]. However, the evaluation of reactive power reserve with respect to contingencies didn't deal with short-term time scale. In this paper, a novel definition of SVRPR is proposed. Voltage support coefficient based on trace sensitivity is adopted to evaluate SVRPR's contribution to short-term voltage stability. To improve the accuracy of evaluation of SVRPR, the second-order trace sensitivity is introduced to the calculation of voltage support coefficient. An optimization model covering multiple contingencies is proposed to improve short-term voltage stability. It is solved by primal dual interior point method.

The rest of this paper is organized as follows: Section 2 introduces the definition and assessment of SVRPR. Section 3 introduces a method of SVRPR optimization. The SVRPR optimization performance of the simulation results on IEEE_39 system are presented in Section 4.

\section{Definitions and Evaluation of SVRPR}

The definitions of SVRPR are established in two-step manner. Generators are the main var sources in power system, in this paper the definition of generator SVRPR is taken as an example. Firstly we propose the definition of SVRPR of individual generator, namely individual SVRPR. The individual SVRPR at different locations in the system should not be directly summed up since they have different contributions to voltage stability. So we define system SVRPR as the sum of weighted individual SVRPR. The weighting factors are decided by individual SVRPR's contribution. The detailed definitions are given as follows.

\subsection{Individual SVRPR}

The generator's automatic excitation system has different properties in short-term and long-term dynamics. In short-term process, it can start forced excitation and produces large amount of reactive power to support system voltage drop. While in long-term process, it is restricted by rated excitation. So reactive support provided by a generator has different properties in short-term and longterm process. On a short-term time scale, the RPR cannot be simply defined as the difference between the maximum reactive power output and the current output because the maximum reactive power output varies with time during transient processes. Thus, we use the following integral to describe the reactive support of generators over a period:

$$
Q_{G R S}=\int_{t_{c}}^{t_{r}} k_{t}\left(Q_{t}(t)-Q_{t 0}\right) \mathrm{dt}
$$

where $Q_{t}(t)$ is the reactive power generator actually provided in the process; $Q_{t 0}$ is the reactive power generator provided before fault. $t_{c}$ is the fault clear time; and $t_{r}$ is the time at the end of the short-term period.

In a transient process, when a generator can provide reactive power more quickly, short-term voltage stability is typically maintained more easily. Different types of generators have different response times to disturbances. To assign larger weights to those generators with faster excitation responses, the attenuating function $K_{t}=e^{-t}$ is used to measure the contribution of the reactive support at different times. In a transient process, promptly providing reactive power plays a key role in voltage support; in addition, the short circuit current attenuates based on the exponential law. Thus, the coefficient accurately reflects the real situation.

This definition can be developed to other dynamic var sources such as Static Var Compensator(SVC) and Static Var Generator(SVG) similarly.

\subsection{System SVRPR}

Short-term voltage stability depends on the level of the system SVRPR, which includes all of the individual SVRPRs. However, the contributions of each individual SVRPR vary based on their locations and dynamics. To assess SVRPR appropriately, a novel definition of the system SVRPR is proposed as follows:

$$
Q_{R S}=\sum_{i=1}^{n_{G}} \int_{t_{c}}^{t_{r}} k_{\mathrm{vs}}(\mathrm{t}) \cdot k_{t}\left(Q_{t}^{i}(\mathrm{t})-Q_{t 0}^{i}\right) \mathrm{dt}
$$

Where $k_{v s}(t)$ is the voltage support coefficient of generator $i$, and $n_{G}$ is the number of generators.

The voltage support coefficient $k_{v s}(t)$ is used to evaluate the individual RPR's contribution. The coefficient is defined as follows: 


$$
k_{v s}(t)=\partial V_{j} / \partial Q_{t}
$$

It corresponds to the voltage sensitivity of weak bus $j$ with respect to $Q_{t}$. This coefficient describes the reactive support capability of generator $i$ to the weak bus $j$. The computation process of $k_{v s}(t)$ will be introduced in detail in the next section. The weak bus is judged by the transient voltage dip acceptable margin $\delta_{v d}$ [16] which is computed by (4):

$$
\delta_{v d}=\left[V_{\min }-\left(V_{c r}-k_{v} T_{c r, v}\right)\right] \times 100 \%
$$

where $V_{\min }$ is the minimum voltage of the relevant bus, $V_{c r}$ is the threshold value of the transient voltage dip, $k_{v}$ is the conversion factor of the critical allowed duration to the voltage deviation, and $T_{c r, v}$ is the critical allowed duration of the bus voltage.

\subsection{Computation of voltage support coefficient based on second-order trajectory sensitivity}

Voltage support coefficient $K_{v s}$ can be expressed as follows:

$$
K_{v s}=\frac{\Delta V_{L}}{\Delta Q_{G}}=\frac{\partial V_{L} / \partial V_{G 0}}{\partial Q_{G} / \partial V_{G 0}}
$$

where $V_{L}$ is voltage of load bus; $Q_{G}$ is reactive power of generator; $V_{G 0}$ is initial voltage of generator bus. To get the value of $K_{v s}, \partial V_{L} / \partial V_{G 0}$ and $\partial Q_{G} / \partial V_{G 0}$ need to be obtained.

Generator reactive power can be expressed as (6).

$$
\mathrm{Q}_{G}=R\left(\mathrm{~V}_{\mathrm{G}}, \delta, \theta, \quad \mathrm{E}_{d}^{\prime}, E_{q}^{\prime}\right)
$$

Then $\partial Q_{G} / \partial V_{G 0}$ can be derived as (7).

$\frac{\partial Q_{G}}{\partial V_{G 0}}=\frac{\partial G R}{\partial E_{d}^{\prime}} \frac{\partial E_{d}^{\prime}}{\partial V_{G 0}}+\frac{\partial R}{\partial E_{q}^{\prime}} \frac{\partial E_{q}^{\prime}}{\partial V_{G 0}}+\frac{\partial R}{\partial V_{G}} \frac{\partial V_{G}}{\partial V_{G 0}}+\frac{\partial R}{\partial \delta} \frac{\partial \delta}{\partial V_{G 0}}+\frac{\partial R}{\partial \theta} \frac{\partial \theta}{\partial V_{G 0}}$

where $V_{G}, \delta, E_{d}^{\prime}, E_{q}^{\prime}$ are voltage magnitude, power angle, direct axis transient field voltage and quadrature axis transient field voltage of generator respectively.

We can see that sensitivity computation of state variables $\left(\delta, E_{d}^{\prime}, E_{q}^{\prime}\right)$ and algebra variables $(\theta, V)$ with respect to generator initial voltage $V_{G 0}$ are included in the computation of $K_{v s}$. So trace sensitivity method is used to compute $K_{v s}$ at different time based on simulation data. The second-order trajectory sensitivity is used to obtain more accurate result. It's computation process is illustrated in appendix. By means of trace sensitivity method, the voltage support coefficient of generator takesinto account the RPR's voltage support to the concerning bus and the RPR's dynamics.

\section{Optimization of SVRPR}

\subsection{SVRPR optimization model}

Based on the definition of SVRPR, the SVRPR optimization model to improve short-term voltage stability is established. In the model, system SVRPR is set as objective to improve short-term voltage stability.

$$
\max \quad Q_{R S}
$$

In practice, multiple contingencies should be covered in the optimization of SVRPR. In such case, we can take the objective as a weighted sum of $\mathrm{Q}_{\mathrm{RS}}$ for each contingency:

$$
\mathrm{Q}_{\mathrm{RS}}=\sum_{j=1}^{N_{f}} \omega_{j} \cdot \mathrm{Q}_{\mathrm{RS}}^{j}
$$

where $\mathrm{Q}_{\mathrm{RS}}^{j}$ is the system SVRPR of contingency $j ; N_{f}$ is the number of severe contingencies; $\omega_{j}$ is the weight of contingency $\mathrm{j}$.

The contingency weight $\omega_{j}$ takes into account both it's probability and severity.

$$
\omega_{j}=-\delta_{v d j} \rho_{j}
$$

where $\rho_{j}$ is the probability of contingency $j$, which is based on the historical fault statistics.

However, the maximization of $Q_{R S}$ is equal to the maximization of $\Delta Q_{\mathrm{RS}}$. And for operators, it is more important to find the direction of control means in time than the optimum control. So the sensitivity of system SVRPR with respect to control variables is linearized so as to reduce computation time. Thus the objective of the model is converted as follows:

$$
\begin{gathered}
\max \left(\Delta \mathbf{Q}_{\mathrm{RS}}\right) \\
\Delta \mathbf{Q}_{\mathbf{R S}}=\frac{\partial \mathbf{Q}_{\mathbf{R S}}}{\partial \mathbf{V}_{\mathbf{G}}} \Delta \mathbf{V}_{\mathbf{G}}+\frac{\partial \mathbf{Q}_{\mathbf{R S} S}}{\partial \mathbf{V}_{S V C \text { ref }}} \Delta \mathbf{V}_{S V C \text { ref }}+\frac{\partial \mathbf{Q}_{\mathbf{R} S}}{\partial \mathbf{V}_{S V G \text { ref }}} \Delta \mathbf{V}_{S V G \text { ref }}
\end{gathered}
$$

where subscripts $S V C$ and $S V G$ indicate var source $S V C$ and $S V G$ respectively.

In this model, control voltage of reactive power source is considered as an optimization variable and the increment of system's reactive power reserve is considered as objective.

The equality constraints of the model correspond to the power flow equations expressed as follows:

$$
\left\{\begin{array}{l}
P_{G i}-P_{L i}-V_{i} \sum_{j=1}^{n} V_{j}\left(G_{i j} \cos \theta_{i j}+B_{i j} \sin \theta_{i j}\right)=0 \\
Q_{G i}-Q_{L i}-V_{i} \sum_{j=1}^{n} V_{j}\left(G_{i j} \sin \theta_{i j}-B_{i j} \cos \theta_{i j}\right)=0
\end{array}\right.
$$

where $P$ and $Q$ mean active and reactive power, $V$ and 
$\boldsymbol{\theta}$ mean bus voltages and angles, subscripts $G$ and $L$ indicate generator and load buses, respectively.

The inequality constraints considered in the model are expressed as follows:

$$
\left\{\begin{array}{l}
V_{i \text { min }} \leq V_{i} \leq V_{i \max }, i=1, \cdots, N \\
V_{S V C g \text { min }} \leq V_{S V C g} \leq V_{S V C g \text { max }}, g=1, \cdots, N_{S V C} \\
V_{S V G h \text { min }} \leq V_{S V G h} \leq V_{S V G h \text { max }}, h=1, \cdots, N_{S V G} \\
Q_{G i \min } \leq Q_{G i} \leq Q_{G i \max }, i=1, \cdots, N_{G} \\
B_{S V C g \text { min }} \leq B_{S V C g} \leq B_{S V C g \max }, g=1, \cdots, N_{S V C} \\
I_{S V G h \text { min }} \leq I_{S V G h} \leq I_{S V G \text { max }}, h=1, \cdots, N_{S V G}
\end{array}\right.
$$

where $V_{S V C g}, V_{S V G h}$ indicate the given voltage of $\mathrm{SVC}_{\mathrm{g}}$ and $\mathrm{SVG}_{\mathrm{h}}$ respectively; $B_{S V C}$ is susceptance of $\mathrm{SVC} ; I_{S V G}$ is the current of SVG; $N, N_{G}, N_{S V C}, N_{S V G}$ are the numbers of buses, generators, SVC and SVG respectively.

\subsection{Solving procedure}

In fact, the converted model is a ORPF problem. It can be solved by PCPDIPM [17]. In this method, the Lagrange multipliers and the slack variables are used to deal with the equality and inequality constraints, and the logarithmic barrier functions are constructed to guarantee the nonnegativity conditions of the slack variables. The Lagrange function without the constraints can be formulated as follows:

$$
L=-\Delta Q_{\mathrm{RS}}-\rho^{T} g(x)+\pi^{T} h_{s}(x)-\mu\left(\sum_{i=1}^{n_{h}} \ln s_{i}\right)
$$

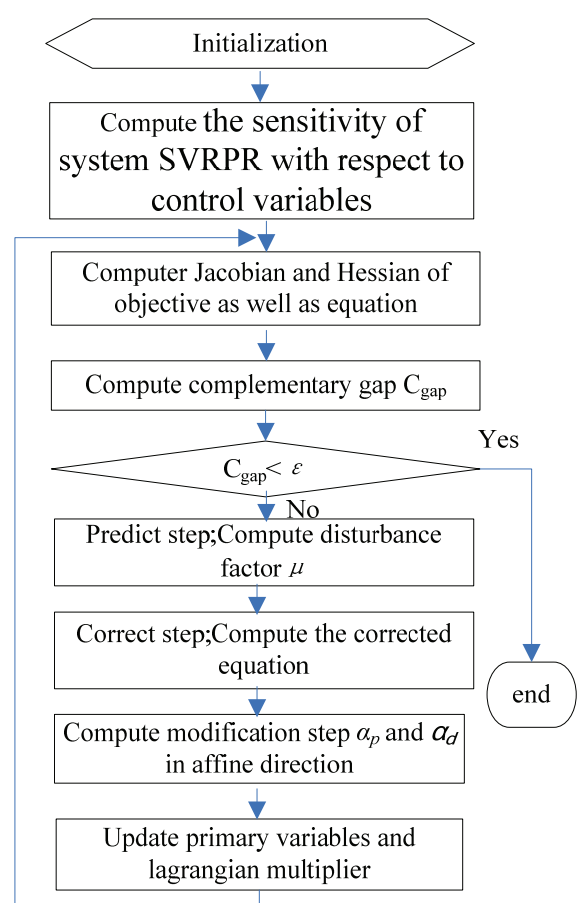

Fig.1. The procedure of optimization based on PDIPM where $g(x)$ represents equality constrains

$h_{s}(\cdot)$ represents inequality constraints with slack variables s.

$\rho$ and $\pi$ are the vectors Lagrange multipliers for the equality and inequality constraints;

$s \quad$ is the vector of slack variables; $\mu$ is the vector of barrier parameters $n_{h}$ is the number of $h_{s}(x)$.

The solving procedure of the optimization is shown as Fig. 1.

\section{Case study}

In this section, the proposed SVRPR optimization model is tested on IEEE 39-bus system. It's line diagram is shown as Fig. 2. Parameters of lines and transformers are from reference [18]. Parameters of generators are listed in Table 1.

For a predefined set of faults, the contingencies studied are the 3-phase symmetric faults at the heads of lines 16-15, 17-16, and 21-16.It is assumed that a fault occurs at $t=0.1$ $\mathrm{s}$ and that the head and terminal breakers of the lines then open successively at $0.09 \mathrm{~s}$ and $0.1 \mathrm{~s}$, respectively. Simulations are carried by PSD-BPA software. The

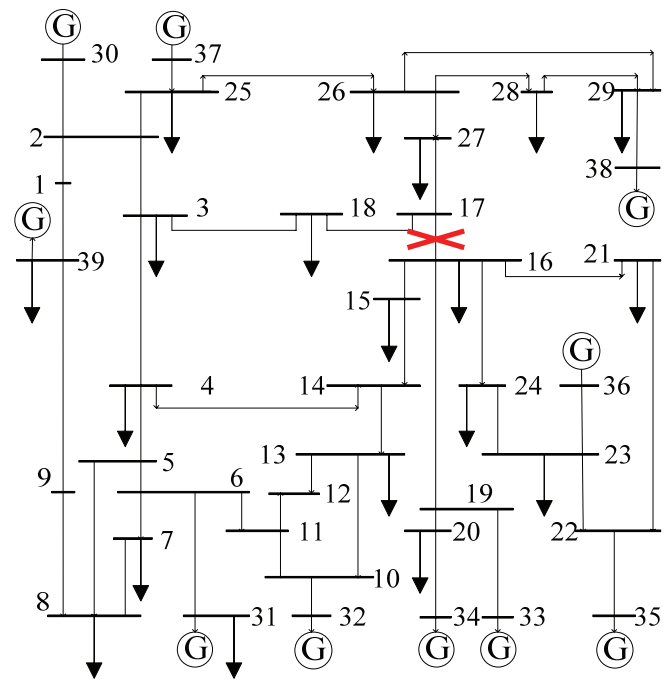

Fig. 2 Structure of IEEE39 system

Table 1. Parameters of generators

\begin{tabular}{ccccccccccc}
\hline Gen. & $\mathrm{E}_{\mathrm{Mws}}$ & $\mathrm{Ra}$ & $\mathrm{Xd}$ & $\mathrm{Xq}$ & $\mathrm{Xd}^{\prime}$ & $\mathrm{Xq}^{\prime}$ & $\mathrm{Td} 0^{\prime}$ & $\mathrm{Tq} 0^{\prime}$ & $\mathrm{Xs}$ & $\mathrm{D}$ \\
\hline 30 & 4200 & 0 & 1 & 0.61 & 0.31 & 0.31 & 10.2 & 1.5 & 0.125 & 0 \\
31 & 3030 & 0 & 2.95 & 2.82 & 0.697 & 0.697 & 6.561 & 1.5 & 0.304 & 0 \\
32 & 3580 & 0 & 2.495 & 2.37 & 0.531 & 0.531 & 5.7 & 1.5 & 0.35 & 0 \\
33 & 2860 & 0 & 2.62 & 2.58 & 0.436 & 0.436 & 5.69 & 1.5 & 0.295 & 0 \\
34 & 2600 & 0 & 6.7 & 6.2 & 1.32 & 1.32 & 5.4 & 0.44 & 0.54 & 0 \\
35 & 3480 & 0 & 2.54 & 2.41 & 0.5 & 0.5 & 7.3 & 0.4 & 0.224 & 0 \\
36 & 2640 & 0 & 2.95 & 2.92 & 0.49 & 0.49 & 5.66 & 1.5 & 0.322 & 0 \\
37 & 2430 & 0 & 2.9 & 2.8 & 0.57 & 0.57 & 6.7 & 0.41 & 0.28 & 0 \\
38 & 3450 & 0 & 2.106 & 2.05 & 0.57 & 0.57 & 4.79 & 196 & 0.298 & 0 \\
39 & 50000 & 0 & 0.2 & 0.19 & 0.06 & 0.06 & 7 & 0.7 & 0.3 & 0 \\
\hline
\end{tabular}


Table 2. Normalized voltage support coefficients of generators

\begin{tabular}{cccccccccc}
\hline \multirow{2}{*}{ fault } & \multicolumn{10}{c}{ Generator } \\
\cline { 2 - 11 } & 30 & 31 & 32 & 33 & 34 & 35 & 36 & 37 & 38 \\
\hline $16-15$ & 0.46 & 0.25 & 0.27 & 0.67 & 0.31 & 0.735 & 0.41 & 0.399 & 0.4 \\
$17-16$ & 0.16 & 0.47 & 0.68 & $\mathbf{1 . 0 0}$ & 0.42 & $\mathbf{0 . 9 1}$ & 0.58 & 0.12 & 0.17 \\
$21-16$ & 0.48 & 0.57 & 0.79 & 0.99 & 0.45 & 0.81 & 0.72 & 0.34 & 0.393 \\
\hline
\end{tabular}

Table 3. Comparison of the transient voltage dip acceptable margin before and after optimization

\begin{tabular}{cccc}
\hline fault & bus & Before optimization $\delta_{v d}$ & After optimization $\delta_{v d}$ \\
\hline $6-15$ from & 17 & $-63.04 \%$ & $12.68 \%$ \\
$17-16$ from & 15 & $-52.42 \%$ & $0.83 \%$ \\
$21-16$ from & 15 & $-68.28 \%$ & $9.85 \%$ \\
\hline
\end{tabular}

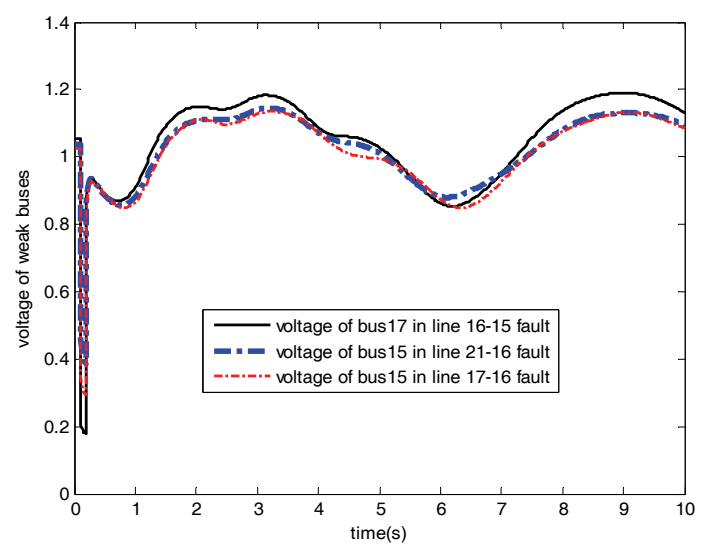

Fig. 3. Multiple fault optimized voltage curves of weak buses

probabilities of all the faults are set as $20 \%$.

As an example, the voltage support coefficients of generators in the fault of line 17-16 are listed in Table 2; these values correspond to the summation of $K_{v s}$ during the transient process and have been normalized. The sensitivity of generator 33 is shown to be the largest, and the sensitivities of generator $30,37,38$, and 39 are far smaller than those of the other generators. The results coincide with the fact that the electric distances between generators $30,37,38$, and 39 and bus 15 become large; thus, their voltage support capacity for bus 15 becomes weaker, as shown in Fig. 2.

Multiple faults considered, the optimized weak bus voltages of the three faults are shown in Fig. 3. From the curves, the voltages of the weak buses are found to all be stable. Concurrently, Table 3 shows that the voltage dips of the weak buses in all three faults have been considerably mitigated. As an example of one single fault, the voltage of bus 15 in the fault of line 17-16 is shown in Fig. 4. However, if only an individual fault is considered, then short-term voltage stability cannot be guaranteed in other faults. For example, for the SVRPR that only includes the fault in line 16-15, the optimized weak bus voltages of the three faults are shown in Fig. 5. The curves
Table 4. Comparsion of transient voltage dip acceptable margin

\begin{tabular}{cccc}
\hline fault & bus & The first-order TS $\delta_{v d}$ & The second-order TS $\delta_{v d}$ \\
\hline $16-15$ from & 17 & $11.98 \%$ & $12.68 \%$ \\
$17-16$ from & 15 & $0.72 \%$ & $0.83 \%$ \\
$21-16$ from & 15 & $9.56 \%$ & $9.85 \%$ \\
\hline
\end{tabular}

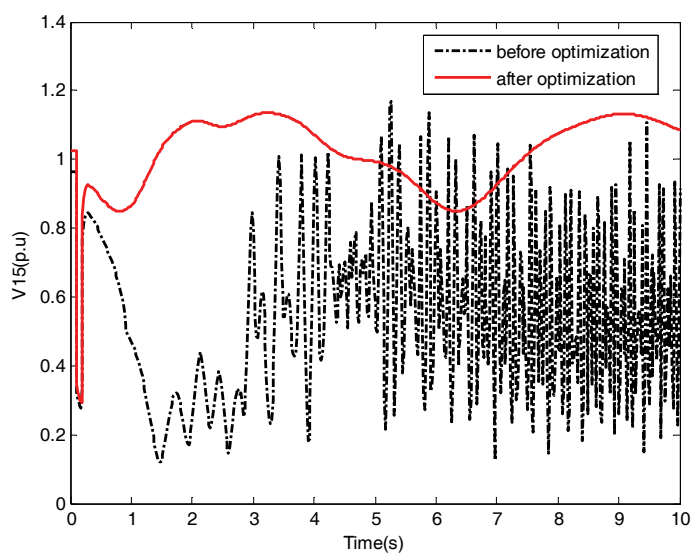

Fig. 4. Voltage curve of bus 15 in line 17-16's head fault

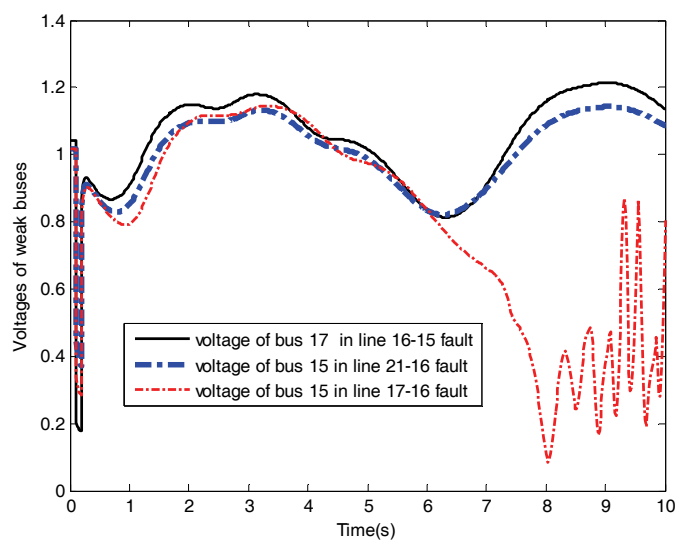

Fig. 5. Single fault optimized voltage curves of weak buses

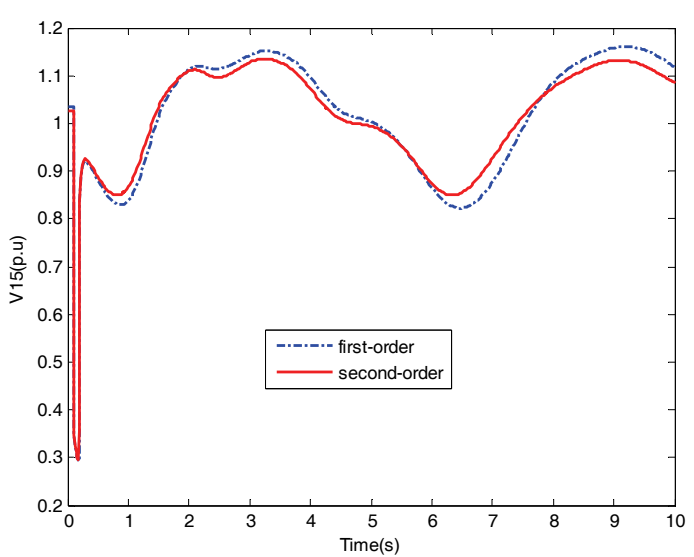

Fig. 6. Optimized voltage curve of bus 15 in line 17-16's fault 
indicate that voltage instability occurs in the fault in line 17-16. It follows that SVRPR optimization with multiple contingencies is essential.

In comparison, we also compute $K_{v s}$ based on the firstorder trace sensitivity. The transient voltage dip acceptable margins of weak buses are listed in Table 4. The improvement is less than using the second-order trace sensitivity. As an example, voltage of bus 15 in line 17-16's head fault is shown in Fig. 6. Comparing the curves, optimization based on the second-order trace sensitivity can improve short-term voltage stability more.

\section{Conclusion}

This paper focuses on a method for reactive reserve optimization to improve short-term voltage stability effecttively. Conclusions of the investigation are summarized as follows:

The definition and three proposed weighting factors of short-term reactive reserve are appropriate and effective. The evaluation method considered both supporting capacity of one bus to the other and demand of reactive power reserve according to severity of faults.

The proposed optimization method of short-term reactive power reserve can improve short-term voltage stability effectively. Compared with the first-order TS, the secondorder TS can indicate the short-term voltage support capability more accurately.

In future research, the scalability of the approach for large-scale power system still needs to be explored. To simplify the computational complexity, the power system is expected be divided into appropriate voltage regions according to short-term voltage response. By this way, short-term voltage problems of each region can be improved respectively with less computation expense.

\section{Acknowledgements}

This research activity is supported by the National Basic Research Program of China (973 Program) under Grant 2014 CB23903.

\section{Appendix}

The second-order trajectory sensitivity is computed as follows:

Differential algebraic equation of power system can be simplified as follows.

$$
\begin{gathered}
\dot{x}=f(x, y, \lambda) \\
0=g(x, y, \lambda)
\end{gathered}
$$

where $x \in\left(\delta, \omega, E_{d}^{\prime}, E_{q}^{\prime}\right), y \in(\theta, V)$ are vectors of state variables and algebraic variables respectively, $\lambda$ is control variable.

By Implicit trapezoidal integration method, differential Eq. (A1) is differenced as (A3).

$$
\mathrm{x}_{k+1}=x_{k}+\frac{\Delta t}{2}\left[f(\cdot)^{k}+f(\cdot)^{k+1}\right]
$$

where $\Delta t$ is the time step of integral.

In every interval $\left(\mathrm{t}^{\mathrm{k}}, \mathrm{t}^{\mathrm{k}+1}\right)$, correction equation of differential algebraic Eq. (A2) and (A3) can be expressed as follows.

$$
\begin{aligned}
& {\left[\begin{array}{cc}
\frac{\Delta t}{2} f_{x}^{k+1}(\cdot)-I \frac{\Delta t}{2} f_{y}^{k+1}(\cdot) \\
g_{x}^{k+1}(\cdot) & g_{y}^{k+1}(\cdot)
\end{array}\right]\left[\begin{array}{l}
\Delta x^{k+1} \\
\Delta y^{k+1}
\end{array}\right]} \\
& =\left[\begin{array}{c}
x^{k+1}-x^{k+1}-\frac{\Delta t}{2}\left[f(\cdot)^{k}+f(\cdot)^{k+1}\right] \\
-g^{k+1}(\cdot)
\end{array}\right]
\end{aligned}
$$

where I is an unit matrix.

The calculation process is mainly divided into two stages: The first-order and second-order trajectory sensitivity.

\section{A. The first-order trajectory sensitivity}

Both the state and the algebraic variables are dependent on the control variable $\lambda$. To calculate the trajectory sensitivity, the sensitivity of the state variables and the algebraic variables to the control variable under steady state must be obtained.

Differential algebraic Eq. (A1) and (A2) can be derivatived as (A5).

$$
\left[\begin{array}{l}
f_{x}(\cdot) f_{y}(\cdot) \\
g_{x}(\cdot) g_{y}(\cdot)
\end{array}\right]\left[\begin{array}{l}
x_{\lambda 0} \\
y_{\lambda 0}
\end{array}\right]=\left[\begin{array}{l}
-f_{\lambda}(\cdot) \\
-g_{\lambda}(\cdot)
\end{array}\right]
$$

Initial values of the first-order trajectory sensitivity can be obtained from (A5). Then (A1) and (A2) are derivatived as (A6) and (A7) in every step.

$$
\begin{gathered}
\mathrm{dx}_{\lambda} / \mathrm{dt}=f_{x}(\cdot) x_{\lambda}+f_{y}(\cdot) y_{\lambda}+f_{\lambda}(\cdot) \\
0=g_{x}(\cdot) x_{\lambda}+g_{y}(\cdot) y_{\lambda}+g_{\lambda}(\cdot)
\end{gathered}
$$

By Implicit trapezoidal integration method, (A6) is differenced as (A8).

$$
\begin{aligned}
0= & {\left[\frac{\Delta t}{2} f_{x}^{k+1}(\cdot)-1\right] x_{\lambda}^{k+1}+\frac{\Delta t}{2} f_{y}^{k+1}(\cdot) y_{\lambda}^{k+1}+x_{\lambda}^{k} } \\
& +\frac{\Delta t}{2}\left[f_{x}^{k}(\cdot) x_{\lambda}^{k}+f_{y}^{k}(\cdot) y_{\lambda}^{k}+f_{\lambda}^{k}(\cdot)+f_{\lambda}^{k+1}(\cdot)\right]
\end{aligned}
$$

Then (A7) and (A8) can be expressed as (A9) in every intervall $\left(\mathrm{t}^{\mathrm{k}}, \mathrm{t}^{\mathrm{k}+1}\right)$. 


$$
\begin{aligned}
& {\left[\begin{array}{cc}
\frac{\Delta t}{2} f_{x}^{k+1}(\cdot)-I \frac{\Delta t}{2} f_{y}^{k+1}(\cdot) \\
g_{x}^{k+1}(\cdot) & g_{y}^{k+1}(\cdot)
\end{array}\right]\left[\begin{array}{l}
x_{\lambda}^{k+1} \\
y_{\lambda}^{k+1}
\end{array}\right]} \\
& =\left[\begin{array}{c}
-x_{\lambda}^{k}-\frac{\Delta t}{2}\left[f_{x}^{k}(\cdot) x_{\lambda}^{k}+f_{y}^{k}(\cdot) y_{\lambda}^{k}+f_{\lambda}^{k}(\cdot)+f_{\lambda}^{k+1}(\cdot)\right] \\
-g_{\lambda}^{k+1}(\cdot)
\end{array}\right]
\end{aligned}
$$

Substituting $\lambda$ with $V_{G 0}$, the first-order trajectory sensitivity of system variables with respect to $V_{G 0}$ can be computed by (A9) in every step.

\section{B. The second-order trajectory sensitivity}

Based on the results of the first-order trajectory sensitivity, the second-order trajectory sensitivity can be further calculated.

Initial values of the second-order trajectory sensitivity can be computed by (A10), which is the derivation of (A5)

$$
\begin{aligned}
& {\left[\begin{array}{l}
f_{x}(\cdot) f_{y}(\cdot) \\
g_{x}(\cdot) g_{y}(\cdot)
\end{array}\right]\left[\begin{array}{l}
x_{\lambda \lambda 0} \\
y_{\lambda \lambda 0}
\end{array}\right]} \\
& =-\left[\begin{array}{c}
f_{x x}(\cdot) x_{\lambda 0}^{2}+2 f_{x y}(\cdot) x_{\lambda 0} y_{\lambda 0}+f_{y y}(\cdot) y_{\lambda 0}^{2}+f_{\lambda \lambda}(\cdot)+f_{x \lambda}(\cdot) x_{\lambda 0}+f_{y \lambda}(\cdot) y_{\lambda 0} \\
g_{x x}(\cdot) x_{\lambda 0}^{2}+2 g_{x y}(\cdot) x_{\lambda 0} y_{\lambda 0}+g_{y y}(\cdot) y_{\lambda 0}^{2}+g_{\lambda \lambda}(\cdot)+g_{x \lambda}(\cdot) x_{\lambda 0}+g_{y \lambda}(\cdot) y_{\lambda 0}
\end{array}\right]
\end{aligned}
$$

In every interval $\left(\mathrm{t}^{\mathrm{k}}, \mathrm{t}^{\mathrm{k}+1}\right)$, the second-order trajectory sensitivity of system variables to the control variable can be obtained by (A11), which is the derivation of (A9).

$$
\begin{aligned}
& {\left[\begin{array}{cc}
\frac{\Delta t}{2} f_{x}^{k+1}(\cdot)-I \frac{\Delta t}{2} f_{y}^{k+1}(\cdot) \\
g_{x}^{k+1}(\cdot) & g_{y}^{k+1}(\cdot)
\end{array}\right]\left[\begin{array}{c}
x_{\lambda \lambda}^{k+1} \\
y_{\lambda \lambda}^{k+1}
\end{array}\right]=-\left[\begin{array}{c}
\alpha \\
g_{\lambda \lambda}^{k+1}(\cdot)
\end{array}\right]}
\end{aligned}
$$

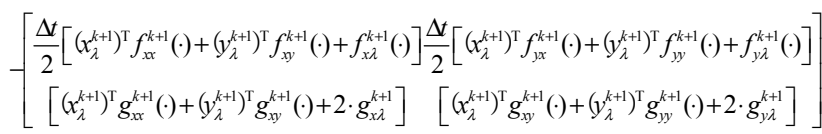

$$
\begin{aligned}
& {\left[\begin{array}{l}
x_{\lambda}^{k+1} \\
y_{\lambda}^{k+1}
\end{array}\right]}
\end{aligned}
$$

where

$$
\begin{array}{r}
\alpha=\frac{\Delta t}{2}\left[\left(x_{\lambda}^{k}\right)^{T} f_{x x}^{k}(\cdot) x_{\lambda}^{k}+\left(y_{\lambda}^{k}\right)^{T} f_{x y}^{k}(\cdot) x_{\lambda}^{k}+\left(x_{\lambda}^{k}\right)^{T} f_{y x}^{k}(\cdot) y_{\lambda}^{k}+\left(y_{\lambda}^{k}\right)^{T} f_{y y}^{k}(\cdot) y_{\lambda}^{k}\right. \\
\left.+f_{x \lambda}^{k}(\cdot) x_{\lambda}^{k}+f_{y \lambda}^{k}(\cdot) y_{\lambda}^{k}+f_{x}^{k}(\cdot) x_{\lambda \lambda}^{k}+f_{y}^{k}(\cdot) y_{\lambda \lambda}^{k}+f_{\lambda \lambda}^{k}+f_{\lambda \lambda}^{k+1}\right]+x_{\lambda \lambda}^{k}
\end{array}
$$

Thus substituting $\lambda$ with $V_{G 0}$, the second-order trajectory sensitivity of state variables with respect to $V_{G 0}$ can be computed by (A11) in every step.

\section{References}

[1] Titare L S, Singh P, Arya L D, et al, "Optimal reactive power rescheduling based on EPSDE algorithm to enhance static voltage stability," International Journal of Electrical Power \& Energy Systems, vol. 63, pp. 588-599, July.2014.

[2] Wu Hao, Guo Ruipeng, et al, "Analysis and computation of effective reactive power reserve of generator," Automation of Electric Power Systems, vol. PS-135, no. 15, pp. 3-17, Aug.2011.

[3] F. Capitanescu and T. Van Cutsem. Evaluation of reactive power reserves with respect to contingencies. in Proc. Bulk Power System Dynamics and Control V, Onomichi, Japan, August 2001.

[4] Mousavi O A, Bozorg M, Ahmadi-Khatir A, et al," Reactive power reserve management: Preventive countermeasure for improving voltage stability margin," in Proceedings of IEEE Power and Energy Society General Meeting, July 2012.

[5] Choi Y H, Seo S, Kang S, et al, "Justification of Effective Reactive Power Reserves With Respect to a Particular Bus Using Linear Sensitivity,” IEEE Trans. Power Systems, vol. PS-26, no.4, pp. 2118-2124, Nov. 2011.

[6] Seo S, Kang S G, Choi Y H, et al, "Verification of effective reactive power reserve with respect to reactive power load demands in the power system," in Proceedings of IEEE Power and Energy Society General Meeting, July. 2010.

[7] Leonardi B and Ajjarapu V, “An Approach for Real Time Voltage Stability Margin Control via Reactive Power Reserve Sensitivities[J]. IEEE Trans on Power Systems, 2013, 28(02) : 615-625.

[8] Arya L D, Singh P and Titare L S, "Anticipatory reactive power reserve maximization using differential evolution," International Journal of Electrical Power \& Energy Systems, vol. PS-35, no. 1, pp. 66-73, Nov. 2012.

[9] Pakari $\mathrm{H}$ and Khajehzadeh A, "Reactive power reserve maximization using intelligent method," CADEMIE ROYALE DES SCIENCES D OUTREMER BULLETIN DES SEANCES, vol. PS-4, no. 2, pp. 245-253, May.2015.

[10] Alizadeh Mousavi O and Cherkaoui R, "Maximum voltage stability margin problem with complementtarity constraints for multi-area power systems," IEEE Trans. Power Systems, vol. PS-29, no. 6, pp. 2993-3002, Mar.2014.

[11] Hwachang S, Byongjun L, Sae-Hyuk K, et al," Reactive reserve-based contingency constrained optimal power flow (RCCOPF) for enhancement of voltage stability margins," IEEE Trans. Power Systems, vol. PS-18, no. 4, pp. 1538-1546, Nov.2003.

[12] Ma Zhaoxing, Chen Hao and Wan Qiulan, “Analysis on Voltage Instability Considering STATCOM Capacity Constraint," Proceedings of the CSEE, vol. PS-33, no. 28, pp. 88-93, Oct. 2013.

[13] Paramasivam M, Dasgupta S, Ajjarapu V, et al, 
"Contingency Analysis and Identification of Dynamic Voltage Control Areas," IEEE Trans. Power Systems, vol. PS-30, no. 6, pp. 2974-2983, Jan.2015.

[14] Li Yikai and Liu Mingbo," Trajectory sensitivity method for transient stability constrained optimal power flow under multi-contingency condition," Proceedings of the CSEE, vol. PS-29, no. 16, pp. 42-48, June.2009.

[15] Chao Zhu and Liu Mingbo, "Transient stability constrained optimal power flow calculation based on second-order trajectory sensitivity," Power System Technology, vol. PS-35, no. 7, pp. 106-112, July.2011.

[16] Xue Y, Xu T, Liu B, et al., "Quantitative assessments for transient voltage security," In Proceedings of the 21st 1999 IEEE International Conference PICA'99. July. 1999.

[17] Balbo A R, Souza M A S, Baptista E C, et al, " Predictor-corrector primal-dual interior point method for solving economic dispatch problems: a post optimization analysis," Mathematical Problems in Engineering, vol. 2012, pp. 1-26, Apr.2012.

[18] Ajjarapu, Venkataramana, ed. Computational techniques for voltage stability assessment and control. Springer, 2007, pp. 241-247.

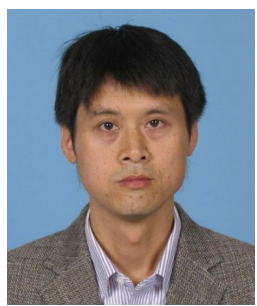

Quancai-Sun He received the B.S and M.S. degree in electrical engineering from Anhui University of Science and Technology, Huainan, China, in 2001 and 2004 respectively. Currently, he is pursuing the Ph.D. degree in electrical engineering at Shanghai Jiao Tong University, Shanghai, China. His research interests include reactive power optimization and voltage stability.

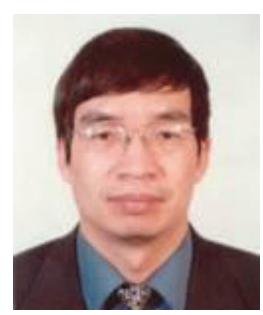

Haozhong Cheng He received the M.S. and Ph.D. degrees in the Department of Electrical Engineering at Shanghai Jiao Tong University, Shanghai, China, in 1986 and 1998, respectively. Currently, he is a Professor of Shanghai Jiao Tong University. His research interests cover power system planning, voltage stability

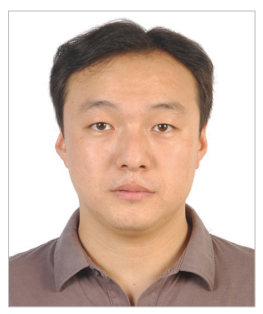

Beijing, China.

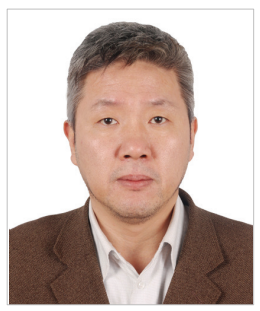

Baiqing Li received the B.S. and M.S. degree from Tsinghua University, and China Electric Power Research Institute, Beijing, China, in 1984, and 1987 respectively. He is a director of power system department, Beijing, China.

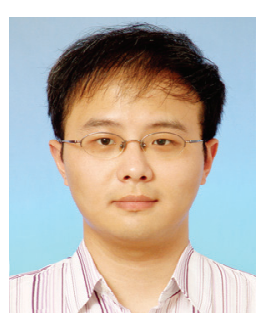

Yue Song He received B.S. and M.S. degrees in electrical engineering from Shanghai Jiao Tong University, Shanghai, China, in 2011 and 2014, respectively. He is currently working toward the $\mathrm{Ph} . \mathrm{D}$. degree in the Department of Electrical and Electronic Engineering, The University of Hong Kong. His research interests include power system stability and optimal reactive power dispatch.

and power quality. 